\title{
The eventual outcome of patients who had lower limb amputations due to peripheral vascular disease at Pelonomi Hospital, Bloemfontein
}

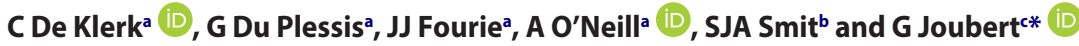 \\ a School of Medicine, Faculty of Health Sciences, University of the Free State, Bloemfontein, South Africa \\ ${ }^{b}$ Department of Surgery, Faculty of Health Sciences, University of the Free State, Bloemfontein, South Africa \\ ' Department of Biostatistics, Faculty of Health Sciences, University of the Free State, Bloemfontein, South Africa
}

\section{*Corresponding author, Email:gnbsgj@ufs.ac.za}

Background: Peripheral vascular disease (PVD) presenting with irreversible lower limb pathology has a high morbidity and mortality rate. This study aimed to determine the outcome of patients who underwent lower limb amputations (LLAs) because of PVD at Pelonomi Hospital, Bloemfontein, 2008-2011.

Methods: Prospective data collection of a retrospective cohort study group was performed. Included were patients with PVDinduced LLAs. Demographic and contact information was collected from Meditech. Living status (alive or dead) was determined using the Department of Home Affairs database and interviews with relatives.

Results: Of the study population $(n=224), 119$ had contact information. Data of 158 people were available to determine living status: $71.5 \%$ had died by follow-up in 2014. Forty-nine interviews were possible, 31 patients had died, with cause of death reported to be primarily vascular related. The cumulative mortality showed that $16.1 \%$ died within a month and $48.4 \%$ within one year post-amputation. Among the 18 living amputees, $50.0 \%$ became unemployed, $77.8 \%$ used wheelchairs, $11.1 \%$ used crutches and $11.1 \%$ a prosthesis.

Conclusion: Lower limb amputation due to PVD is indicative of a poor prognosis. In our study only $28.5 \%$ of patients survived 3-6 years post-amputation. Restoration to ideal mobility was achieved in a minority of cases.

Keywords

Bloemfonteinlower limb amputationspatient outcomeperipheral vascular disease

\begin{abstract}
Introduction
Peripheral vascular disease (PVD) is reported to be the most common cause of major amputation worldwide. ${ }^{1}$ Peripheral vascular disease presenting with irreversible lower limb pathology is known to have a high rate of morbidity and mortality. ${ }^{2}$
\end{abstract}

In a Dutch study, Fortington et al. ${ }^{3}$ reported a 30-day mortality rate of $22 \%$, one-year mortality of $44 \%$ and five-year mortality of $77 \%$ in a cohort of 299 patients who underwent a first-time lower limb amputation. Cardiovascular and renal disease significantly increased the odds of dying. A study ${ }^{4}$ in the United Kingdom on 339 patients undergoing lower limb amputation for PVD or complications due to diabetes reported mortality rates of $12.4 \%$ at 30 days and $35.7 \%$ at one year, with age, ASA status, out-ofhours surgery and renal disease being associated with mortality. In a United States study ${ }^{5}$ on more than 186000 patients with peripheral artery disease older than 65 years undergoing major lower limb amputation, mortality rate was reported as $13.5 \%$ at 30 days and $48.3 \%$ at one year. Age, history of heart failure, renal disease and chronic obstructive pulmonary disease were significantly associated with mortality. A study in Johannesburg ${ }^{6}$ ( $n=73$ ) found that older age, and smoking and alcohol use were associated with mortality at three months after lower limb amputation.

Norgren et al. ${ }^{2}$ reported the outcome of patients two years after they had undergone a below-knee amputation (BKA) as $40 \%$ having regained full mobility while $15 \%$ had a subsequent above-knee amputation (AKA), 15\% had an additional contralateral amputation and $30 \%$ had passed away.

Our study was motivated by subjective observations that the follow-up visits of amputees to our clinics were infrequent and erratic.

\begin{abstract}
Aim of the study
The aim of the study was to determine the outcome of patients who had undergone PVD-induced lower limb amputations at Pelonomi Hospital, Bloemfontein. Mortality was compiled as the cumulative mortality within a predetermined timeframe. If possible, the cause of death was also determined: either to be vascular or non-vascular related. In addition, the outcome as to functionality and mobility was determined with interviews conducted with the patient or family involved.
\end{abstract}

\section{Materials and methodology}

\section{Study design}

This was a retrospective cohort study with prospective data collection.

\section{Patient Characteristics}

Included in the study were all patients who had PVD-induced 


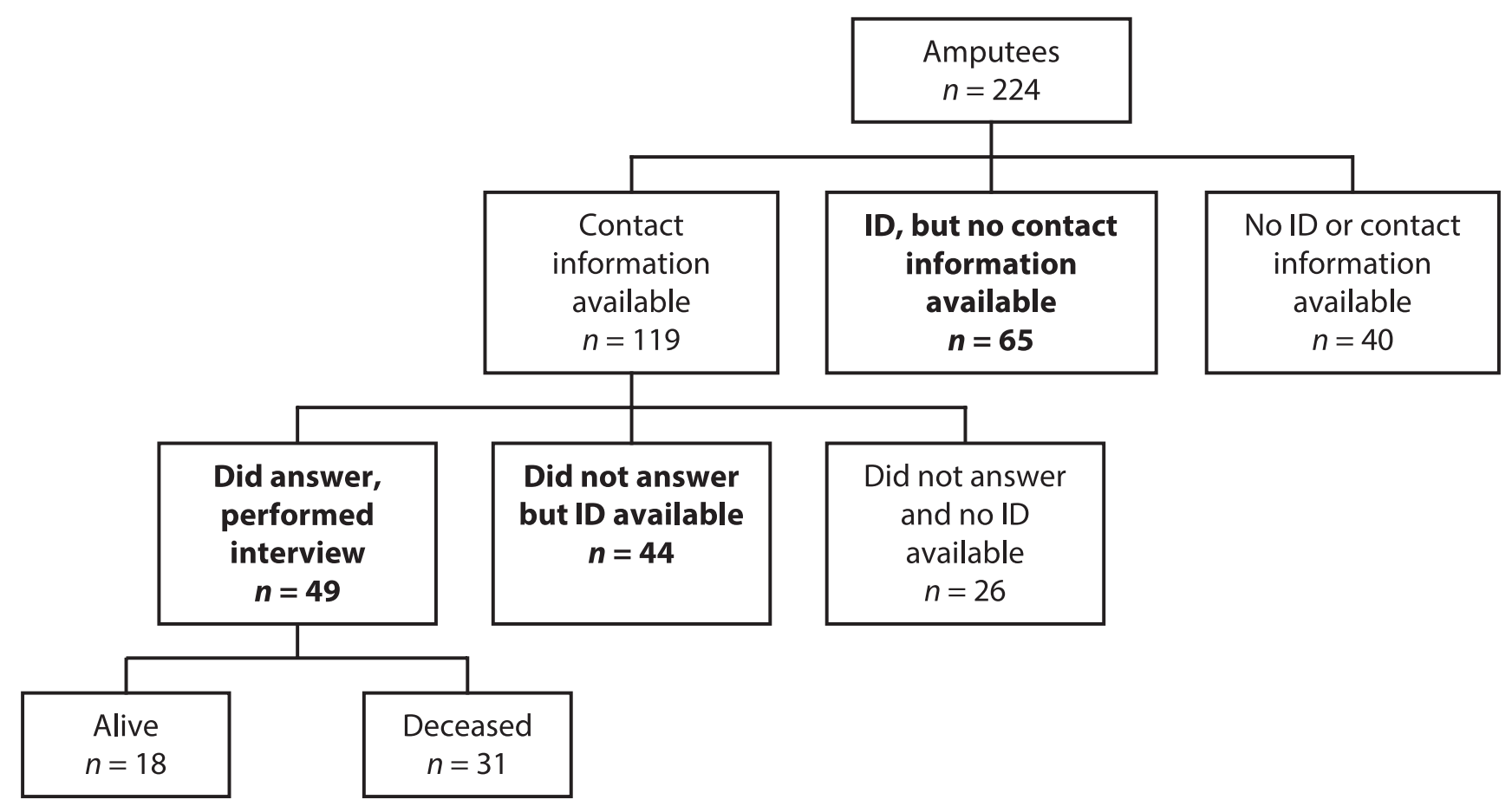

Figure 1: Breakdown of patients with lower limb amputation during the study period January 2008 to December 2011. Note: *Blocks indicated in bold are those patients for whom mortality information could be obtained.

lower limb amputations, including BKA or AKA from January 2008 to December 2011.

\section{Measurements}

In 2014, theatre lists of all surgeries between January 2008 and December 2011 at the Departments of Surgery and Orthopaedics were provided by Pelonomi Hospital. The lists contained information on patient numbers and procedures performed. The patient numbers of those with lower limb amputation were entered into the Meditech database to obtain electronic medical records, contact information and basic demographic data.

Patients were assigned unique study numbers and relevant data were captured on data forms. Data collected included patient profile (age and gender) and status of the patient (alive or dead). If the patient was deceased, the time and possible cause of death were recorded. If the patient was alive, mobility of the patient

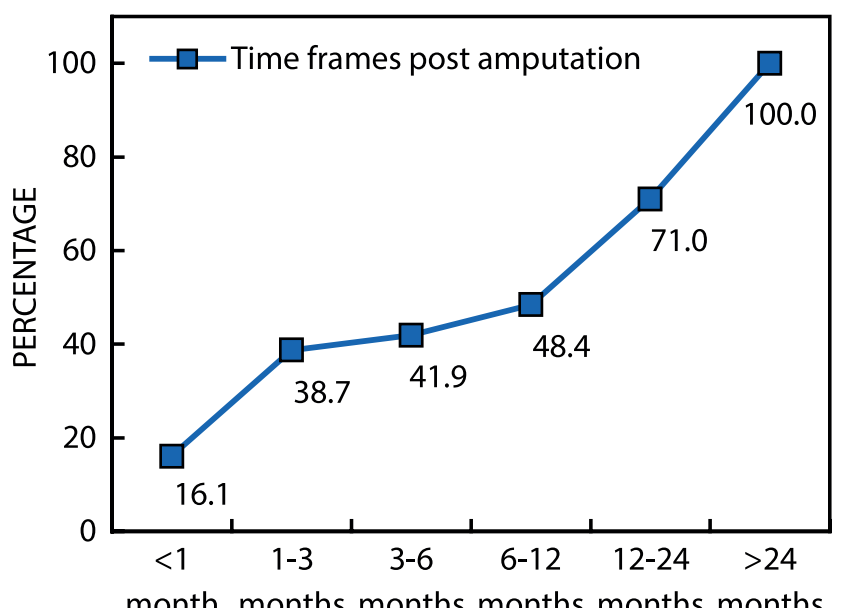

Figure 2: Cumulative mortality over a two-year period post-amputation $(\boldsymbol{n}=31)$. (whether walking with prosthesis or a crutch, bedridden or in a wheelchair) as well as a change in employment history were recorded.

Patients with available contact information were phoned by the research assistant for the purpose of scheduling an interview. To determine living status (alive or dead), the identity (ID) numbers of patients (when available) were entered into the database of the Department of Home Affairs. ${ }^{7}$

A pilot study was performed using 10 randomly selected patients with lower limb amputation to test the study methodology. No changes had to be made. The results obtained during the pilot study were included in the main study.

\section{Ethical considerations}

The study protocol was approved by the Ethics Committee of the Faculty of Health Science, University of the Free State (UFS). Permission to access patient records was given by the Clinical Head of Pelonomi Hospital. Participation in the interviews by either the patients or the next of kin was voluntary. Detailed information regarding the study and participation was provided by the research assistant and thus informed verbal consent was obtained.

\section{Data analysis}

Results were summarised by frequencies and percentages (categorical variables) and means, standard deviations or percentiles (numerical variables). Analysis was performed by the Department of Biostatistics, UFS.

\section{Results}

\section{Study population}

The study population consisted of 224 patients. The age of the 
patients ranged from 24 to 93 years with a median age of 64 years. The population consisted of $42.4 \%$ female and $57.6 \%$ male patients. Patients with AKAs represented $46.9 \%$ of the study population compared with $53.1 \%$ BKAs.

The follow-up of the study population is depicted in Figure 1. For only $49(21.9 \%)$ patients was an interview possible. For 109 patients ( $n=44+65,48.7 \%$ ) the database of the Department of Home Affairs was the only available source to determine living status.

The response rate of patients $(n=119)$ with contact information per study year was $52.3 \%$ (2008), $75.0 \%$ (2009), $48.1 \%$ (2010) and $51.6 \%(2011)$.

\section{Mortality}

Data (ID and/or interviews) of 158 people ( $n=65+44+49$, see Figure 1) were available to determine living status. Of those patients, 113 (71.5\%) had died by the time of follow-up in 2014. The percentage who had died by 2014 per year of amputation was as follows: 2008 85.3\%, 2009 80.0\%, 2010 70.5\% and 2011 $63.3 \%$. From the 49 interviews with the patients or next of kin, it was found that 31 patients had died. Figure 2 shows that for these 31 patients mortality was $16.1 \%$ within a month and $48.4 \%$ within one year after the amputation, cumulatively. Causes of death $(n=31)$ were reported to be $71.0 \%$ vascular related, $12.9 \%$ non-vascular related and $16.1 \%$ unknown.

\section{Living amputees}

Under this category only 18 interviews were available: $50.0 \%$ ( $n=9$ ) patients lost their work after the amputation, of whom seven patients regarded the amputation as the cause of their unemployment. None of them were later re-employed. The majority of the 18 patients $(77.8 \%)$ used wheelchairs and the remaining $22.2 \%$ were divided equally between either using crutches or walking with a prosthesis. No one was bedridden.

\section{Discussion}

In this study, no evidence of an ID number or any contact details could be found in the files of $17.8 \%$ of the population that underwent lower limb amputation. Incorrect and invalid data not only hamper treatment efforts, but may negatively affect the patient.

It was expected that the telephone response rate would increase over time due to greater availability and affordability of cell phones. However, the response rate was erratic. Of those with available contact numbers only $41.2 \%$ could be reached. It is unclear how the large number of untraceable patients would affect the results. Given that $71.5 \%$ of the study population had died by follow-up in 2014, it may be possible that most of the patients with contact numbers but not responding to calls had passed away.

This study population had a median age of 64 years, which is older than the mean of 52.9 years for surviving and 58.5 years for the non-surviving patients reported in Johannesburg, ${ }^{6}$ but younger than the mean of 74.1 years and median of 73 years reported by Fortington et al. ${ }^{3}$ and Scott et al., ${ }^{4}$ respectively. Males were in the majority in our study $(57.6 \%)$, as was the case in the above three studies, which reported the percentage of males ranging from $60 \%$ to $70 \%$ of patients. ${ }^{3,4,6}$ Norgren et al. ${ }^{2}$ reported that the prevalence of PVD is slightly greater in men.

In our study, the follow-up mortality rate of $71.5 \%$ post- amputation was high. Fortington et al. ${ }^{3}$ reported $77 \%$ of patients having died by five years post-amputation, compared with our figure for amputations conducted in 2009 of $80.0 \%$. Norgren et $a l^{2}$ reported the mortality rate in patients with diagnosed critical limb ischaemia as $55 \%$ at five years and $90 \%$ at 10 years. The mortality rate in the study by Nehler et al. ${ }^{8}$ was $45 \%$ at three years compared with our figure of $63.3 \%$ for amputations performed in 2011.

Information regarding date of death and cause of death could only be determined telephonically by interviews with next of kin and not via the Department of Home Affairs database. These selfreported data must be viewed with caution. The majority of deaths were reported to be vascular related. Irreversible vasculopathy of the lower limb affects vital organs in a similar way. Norgren et al. $^{2}$ stated that coronary artery disease is by far the most common cause of death among patients with PVD (40$60 \%$ ). Cerebral artery disease accounted for $10-20 \%$ of the deaths; other vascular events such as ruptured aortic aneurism caused approximately $10 \%$ of deaths.

Other factors, apart from vascular disease, could play a part in the high mortality. Due to their age, these patients are prone to age-related diseases. Other factors that were not measured in this study include physical impairment with the resulting loss of income, which may have led to poor socio-economic conditions, and unavailability or physical challenges of using a wheelchair or prosthesis, which may have resulted in a sedentary lifestyle with associated morbidity and mortality.

The majority of living amputees who had lost their jobs blamed it on the amputation. None of them were re-employed; however, this could be expected considering their median age of 64 years.

Nehler et al. ${ }^{8}$ found that among the surviving patients (at 17 months) $46 \%$ were non-ambulatory. Information from only $8 \%$ of the study population was available to determine the method of mobility in our study. This response rate was too low to make a reliable conclusion as to the true state of mobility.

Norgren et $a^{1}$ have reported that PVD is 'under-recognised by physicians ... suboptimally treated' and that the public also has poor knowledge regarding this condition. The impact of these factors in our setting needs investigation.

\section{Conclusion}

Lower limb amputation due to PVD is indicative of a poor prognosis. In our study only $28.5 \%$ of lower limb amputees survived three to six years post-amputation. Restoration to ideal mobility was achieved in a minority of cases.

\section{Recommendations}

It is imperative that cardiovascular risk factors of amputees should be addressed aggressively. It is equally important that amputees are supported with a comprehensive mobilisation and rehabilitation programme.

Acknowledgements - The authors would like to thank Mrs E. Martins, research assistant at Department of Surgery, UFS, for doing the telephone interviews as well as Mr F.C. van Rooyen, Department of Biostatistics, Faculty of Health Sciences, University of the Free State, for statistical assistance.Ms T. Mulder, medical editor, School of Medicine, University of the Free State, is thanked for technical and editorial preparation of the manuscript. 


\section{ORCID}

C De Klerk (D) http://orcid.org/0000-0002-9233-1012

A O'Neill (D) http://orcid.org/0000-0003-2211-5637

G Joubert (D) http://orcid.org/0000-0002-3728-6925

\section{References}

1. Norgren L, Hiatt WR, Dormandy JA, et al. The next 10 years in the management of peripheral artery disease: perspectives from the 'PAD 2009' conference. Eur J Vasc Endovasc Surg. 2010;40(3):375-80. doi: http://dx.doi.org/10.1016/j.ejvs.2010.05.005.

2. Norgren L, Hiatt WR, Dormandy JA, et al. Inter-society consensus for the management of peripheral arterial disease (TASC II). Eur J Vasc Endovasc Surg. 2007;33(1):S1-S75. doi: http://dx.doi.org/10.1016/j.ejvs.2006.09.024.

3. Fortington LV, Geertzen JH, van Netten JJ, et al. Short and long term mortality rates after a lower limb amputation. Eur J Vasc Endovasc Surg. 2013;46(1):124-31. doi: http://dx.doi.org/10.1016/j.ejvs.2013.03.024.

4. Scott SW, Bowrey $S$, Clarke $D$, et al. Factors influencing short- and long-term mortality after lower limb amputation. Anaesthesia. 2014;69(3):249-58. doi: http://dx.doi.org/10.1111/anae.12532.

5. Jones WS, Patel MR, Dai D, et al. High mortality risks after major lower extremity amputation in Medicare patients with peripheral artery disease. Am Heart J. 2013;165(5):809-15.e1. doi: http://dx.doi.org/10.1016/j.ahj.2012.12.002.

6. Godlawa L, Steward A, Musenge E. Differences in characteristics between people with lower limb amputations who died before 12 weeks and those who survived: short report. S Afr J Physiotherapy. 2011;67(1):44-7.

7. Department of Home Affairs, South Africa. Available from: http:// www.dha.gov.za.

8. Nehler MR, Coll JR, Hiatt WR, et al. Functional outcome in a contemporary series of major lower extremity amputations. J Vasc Surg. 2003;38(1):7-14. doi: http://dx.doi.org/10.1016/S0741-5214(03)00092-2. 